\title{
ELABORAÇÃO DE GELEIAS MISTAS, NAS FORMULAÇÕES TRADICIONAL, LIGHT E DIET A PARTIR DA CASCA DO MARACUJÁ AMARELO (PASSIFLORA EDULIS FLAVICARPA DEGENER) - Jun.2011
}

\section{PREPARATION OF MIXED JELLIES, FORMULATIONS IN TRADITIONAL DIET AND LIGHT FROM THE BARK OF PASSION FRUIT (Passiflora edulis flavicarpa Degener) - Jun.2011}

\author{
Cinthia Marcela Rodrigues Silva ${ }^{1}$; Maíra Aparecida Mangaba ${ }^{2}$; Flávia Maria Vasques Farinazzi-Machado ${ }^{3}$; \\ Elke Shigematsu ${ }^{4}$ \\ ${ }^{1}$ FATEC - Marília - São Paulo - Brasil cinthia_moria@ hotmail.com \\ ${ }^{2}$ FATEC - Marília - São Paulo - Brasil maira_mbg@ hotmail.com \\ ${ }^{3}$ FATEC - Marília - São Paulo - Brasil farinazzimachado@ hotmail.com \\ ${ }^{4}$ FATEC - Marília - São Paulo - Brasil elke_ds@ hotmail.com
}

\begin{abstract}
Resumo
No setor alimentício partes da matéria-prima não utilizadas ou descartadas durante o processamento são denominadas resíduos. O processamento do suco industrializado de maracujá gera toneladas de cascas e sementes que podem ser utilizados na elaboração de diversos subprodutos como geleias. Este trabalho teve como objetivo, a elaboração de geleias mistas de abacaxi com maracujá, incluindo sua casca, em diferentes formulações (tradicional, light e diet), visando o aproveitamento de resíduos de indústrias de alimentos além de verificar a aceitabilidade do produto. As geleias foram elaboradas no Laboratório de Processamento de Alimentos da Faculdade de Tecnologia de Marília-SP. Foram preparadas utilizando abacaxi da cultivar hawai (Smooth Cayenne), maracujá amarelo (Passiflora edulis Flavicarpa Degener), sacarose, xarope de glicose e edulcorantes, obtendo valores finais de sólidos solúveis de $65^{\circ}$ Brix para tradicional e valores entre $48^{\circ}$ Brix para light e $43,9^{\circ}$ Brix para diet. Avaliaram-se as características físicoquímicas e sensoriais. Todas as formulações apresentaram um índice de aceitabilidade maior que 78\%. Quanto ao teor de fibras encontrado é possível indicar sua ingestão na prevenção de doenças gastrointestinal, sendo viável a comercialização de geleias tradicional, light e diet utilizando o albedo do maracujá em substituição à pectina comercial.
\end{abstract}

Palavras-chave: reaproveitamento de resíduos; fibras; novos produtos; abacaxi; sólidos solúveis.

\section{Introdução}

No setor alimentício, partes da matéria-prima não utilizadas ou descartadas durante o processamento são denominadas resíduos, que na maioria das vezes são mais nutritivas do que outras partes do alimento que estamos habituados a ingerir (EVANGELISTA, 2008; BADAWI, 2010). 
Durante a elaboração de determinados produtos ocorrem perdas significativas, porém, a utilização desses resíduos resultaria em diminuição de custos e gastos energéticos, sendo o desconhecimento de como reaproveitá-los um dos fatores principais para o desperdício. Uma boa alternativa para evitá-lo seria a utilização desses resíduos como fonte de fibras, além de aumentar seu valor comercial (KROLOW, 2005; SOUZA et al., 2008; OLIVEIRA et al., 2002).

O processamento do suco industrializado de maracujá gera toneladas de resíduos como cascas e sementes, (FIGUEIREDO et al., 2009) que podem ser utilizados na elaboração de diversos subprodutos na alimentação humana e animal, por exemplo, na fabricação de geleias (EVANGELISTA, 2008; ISHIMOTO et al., 2007; SALINAS, 2002; PINHEIRO, 2007; GIUNTINI et al., 2003).

A casca do maracujá apresenta grande quantidade de vitaminas, minerais, proteínas e pectina, sendo que suas concentrações variam de acordo com o estágio de maturação do fruto. A pectina é uma fibra solúvel que dificulta a absorção de carboidratos e gorduras pelo organismo, reduz a taxa glicêmica e os níveis de LDL-c (CÓRDOVA et al., 2005; ROCKENBACH, 2007; GAVA, 2002; CAMARGO et al., 2007). No organismo, a pectina do albedo auxilia no esvaziamento gástrico e no trânsito intestinal, pois retém água formando géis viscosos (CASTRO e FRANCO, 2002).

O abacaxi destaca-se pelo valor energético, devido à sua alta composição de açúcares, e valor nutritivo pela presença de sais minerais (cálcio, fósforo, magnésio, potássio, sódio, cobre e iodo) e de vitaminas (C, A, B1, B2 e Niacina), além da bromelina que auxilia no processo de digestão. Mesmo com baixo teor de pectina, o abacaxi é adequado para a fabricação de geleias devido ao seu teor de ácido (GRANADA et al., 2004).

Atualmente, a busca por alimentos mais saudáveis e de baixo valor calórico tem sido estudada para pesquisa e desenvolvimento de novos produtos a base de frutas e derivados que além de nutrir auxiliam na prevenção e controle de doenças como, diabetes, obesidade, hipertensão ou mesmo preocupação com a estética corporal. A proposta da indústria de alimentos é desenvolver esses produtos sem comprometimento do sabor, sendo esta melhora possível devido a tecnologia aplicada e melhoria dos ingredientes, como por exemplo, o desenvolvimento de produtos diet e light (GRANADA et al., 2005; SPANHOLI e OLIVEIRA, 2009; MAIA, 2007; DERIVI e MENDEZ, 2001).

No entanto, a população, de modo geral, não tem conhecimento sobre a diferenciação sobre estes termos e o correto uso destes produtos. E as informações contidas na embalagem, muitas vezes, não são claras ao entendimento do consumidor (ORIANI, 2010).

Produtos light possuem redução, de no mínimo $25 \%$, de um determinado nutriente ou teor calórico, com finalidades para dietas e controle de peso. Já os produtos nomeados diet devem ser 
isentos ou conter no máximo $0,5 \%$ de açúcares na sua composição, sendo estes utilizados de acordo com a legislação brasileira para dietas diferenciadas e/ou opcionais, auxiliando indivíduos em condições metabólicas e fisiológicas específicas (FREITAS, 2005).

Seguindo tendências do mercado consumidor, várias pesquisas têm sido desenvolvidas para a obtenção de geleias light e diet. Nesses tipos de geleias, o açúcar é substituído por edulcorantes, como ciclamato, sacarina, acesulfame-K, estevesídeo, aspartame e sucralose, associados a um agente de corpo maltitol ou sorbitol (GRANADA et al., 2005; ORTOLANI et al., 2008).

Este trabalho teve como objetivo, a elaboração de geleias mistas de abacaxi com maracujá, incluindo sua casca, em diferentes formulações (tradicional, diet e light), visando o aproveitamento de resíduos de indústrias de alimentos além de verificar a aceitabilidade do produto.

\section{Material e Métodos}

Utilizou-se para elaboração das geleias, abacaxi da cultivar hawai (Smooth Cayenne), maracujá amarelo (Passiflora edulis Flavicarpa Degener), sacarose e edulcorante sucralose da marca Splenda, adquiridos no comércio local, xarope de glicose da empresa Cargill, além do maltitol, fornecido pela empresa DietMax.

As geleias tradicional e light foram elaboradas com uma mistura de polpas de maracujá e abacaxi, sendo utilizadas nas formulações, concentrações de $40 \%$ e $20 \%$ de sacarose, respectivamente. Para a geleia diet utilizou-se $40 \%$ de uma mistura de edulcorantes sucralose e maltitol adicionada a mistura de polpas, de acordo com o cálculo da intensidade de doçura (CÂNDIDO e CAMPOS, 1996).

\section{Elaboração de geleias}

Realizou-se a elaboração de geleias mistas de abacaxi com maracujá em diferentes formulações no Laboratório de Processamento de Alimentos da Faculdade de Tecnologia de Marília.

Primeiramente, as frutas inteiras passaram por um processo de limpeza e sanitização em água clorada a 200 ppm por 15 minutos, em seguida realizou-se a pesagem. Logo após, o abacaxi passou pelas operações manuais de descascamento e corte da polpa; os maracujás passaram por processos manuais de corte central e retirada da polpa. Posteriormente, as cascas foram colocadas em processo de cocção com monitoramento de temperatura, para que esta não ultrapassasse $90{ }^{\circ} \mathrm{C}$. Após aproximadamente vinte minutos de cocção, as cascas do maracujá foram retiradas do processo 
térmico, e posteriormente retirou-se o albedo (parte branca), que foi adicionado de água e triturado no liquidificador, e por fim, peneirado.

A polpa do abacaxi foi acrescentada água e misturada em liquidificador até obtenção do suco, de $12{ }^{\circ}$ Brix, e peneirado.

Para elaboração de geleia tradicional, misturou-se o suco de abacaxi com a polpa do maracujá incluindo as sementes. Adicionou-se sacarose até completa homogeneização com a polpa e suco, em seguida iniciou-se o processo de cocção, e por fim acrescentou-se a casca do maracujá (albedo) em substituição à pectina comercial até atingir $65^{\circ}$ Brix. Nas formulações diet e light, seguiram-se o mesmo procedimento, substituindo a sacarose pelos edulcorantes sucralose e maltitol, com concentrações de sólidos solúveis a 43,9 e $48^{\circ}$ Brix, respectivamente.

As geleias foram envasadas em frascos de vidro esterilizados a $100{ }^{\circ} \mathrm{C}$ por 15 minutos e fechadas com tampa de metal para formação de vácuo em seu interior, em seguida, invertidas, por cinco minutos, resfriadas e armazenadas em temperatura ambiente.

\section{Análise da Composição Centesimal}

Avaliaram-se as três formulações de geleias quanto ao teor de umidade, cinzas, lipídeos, proteínas, fibras totais e carboidratos de acordo com os métodos preconizados pelo Instituto Adolfo Lutz (2005).

\section{Análises Físicas e Químicas}

As três formulações de geleia de abacaxi com maracujá foram analisadas quanto ao $\mathrm{pH}$, acidez titulável (\% ácido cítrico) e sólidos solúveis totais ( ${ }^{\circ}$ Brix) de acordo com os métodos preconizados pelo Instituto Adolfo Lutz (2005).

\section{Análise Sensorial}

A análise sensorial foi realizada com 50 provadores não treinados, de ambos os gêneros, período noturno do curso Técnico em Administração da Escola Técnica Antônio Devisate, localizada na cidade de Marília-SP.

Utilizou-se o método de análise escala hedônica estruturada de nove pontos, sendo um (desgostei muitíssimo) e nove (gostei muitíssimo) segundo Gularte (2002), para avaliação da aparência, aroma, sabor, textura e impressão global, juntamente com questionário sócio econômico abrangendo os itens: sexo, faixa etária, renda e grau de instrução. 
Sendo os cálculos utilizados:

AS $(\%)=$ soma das percentagens globais das notas 6 à 9 na escala hedônica.

$\mathrm{IA}(\%)=\mathrm{A} * 100 / \mathrm{B}$

A- média obtida

B- nota mais alta obtida na escala.

As amostras foram apresentadas aos provadores em copos descartáveis brancos, codificados com três algarismos aleatórios. Cada amostra foi constituída por cerca de $20 \mathrm{~g}$ de geleia, à temperatura ambiente, sendo orientado aos provadores que ingerissem água durante o intervalo de cada amostra, para limpeza das papilas.

\section{Análise Estatística}

Os resultados foram avaliados pela análise de variância (ANOVA), ao nível de 5\% de significância utilizando o software Bioestat, envolvendo médias, desvio padrão e análise de variância das notas atribuídas.

\section{Resultados e discussão}

\section{Composição Centesimal}

Os resultados das análises de composição centesimal das geleias tradicional, light e diet estão descritos na Tabela 1.

Tabela 1 - Resultados da composição centesimal das diferentes formulações de geleias

\begin{tabular}{cccc}
\hline & Tradicional & Light & Diet \\
\hline Valor Energético (kcal/100g) & 201,3 & 146,7 & 113,4 \\
Umidade (g) & 25,8 & 43,4 & 48,2 \\
Cinzas (g) & 0,4 & 0,4 & 0,4 \\
Lipídeos (g) & 0,1 & 0,4 & 0,1 \\
Carboidratos (g) & 48,8 & 32,5 & 25,7 \\
Proteínas (g) & 1,3 & 3,2 & 2,3 \\
Fibra bruta (g) & 23,5 & 20,0 & 23,1 \\
\hline
\end{tabular}

Os menores valores para proteínas e lipídeos foram encontrados na formulação tradicional, diferentemente os valores de carboidratos foram maiores devido a utilização de sacarose e da matéria prima empregada. De acordo com Franco (1993) o maracujá é rico em açúcares e, em grande parte, de glicose e frutose.

Nas geleias light e diet os açúcares totais apresentaram valores entre $32,47 \mathrm{~g}$ e $25,71 \mathrm{~g}$, sendo este menor quando comparado ao estudo realizado por (NACHTIGAL, 2004) na elaboração de 
geleias light de amora preta em diferentes concentrações de sacarose, onde obteve resultados entre $33,36 \mathrm{~g}$ a $36,73 \mathrm{~g}$.

Os valores calóricos para as geleias light e diet encontrados em relação a tradicional apresentaram redução de $27,33 \%$ e $43,61 \%$, respectivamente, visto que houve substituição de açúcares por edulcorantes alternativos de baixo valor calórico, nestes produtos.

As formulações tradicional e diet não diferiram significativamente quanto ao teor de fibras, já a light teve uma redução de 14,88\% quando comparada a amostra tradicional. A Agência Nacional de Vigilância Sanitária (ANVISA) determina que para um produto ser considerado como fonte de fibras, este deve conter $3 \mathrm{~g}$ em $100 \mathrm{~g}$ da porção em sua formulação. Comparando os produtos formulados neste trabalho os teores de fibras foram maiores em relação ao estudo desenvolvido por Lamante et al.(2005), este encontrou $5 \mathrm{~g} / 100 \mathrm{~g}$ em geleia diet de maracujá utilizando aspartame e estévia, o mesmo ocorreu no desenvolvimento de geleia de goiaba diet enriquecida com fibras elaborada por Lazarin et al.(2011), obtendo 3,08g/100g de fibras totais.

\section{Análise físicas, químicas e bioquímicas}

Observou-se através dos resultados que a formulação diet apresentou o menor valor de $\mathrm{pH}$, porém a diferença foi considerada mínima entre as amostras, como pode ser notado na Tabela 2.

Tabela 2 - Determinações físicas, químicas e bioquímicas das diferentes formulações de geleias

\begin{tabular}{cccc}
\hline & Tradicional & Light & Diet \\
\hline $\mathrm{pH}$ & 3,3 & 3,3 & 3,2 \\
Acidez titulável (\%) & 14,6 & 15,8 & 17,0 \\
\% sólidos solúveis (Brix) & 65 & 48 & 43,9 \\
\hline
\end{tabular}

Segundo Lago et al. (2006) sugere-se para geleias, um pH máximo de 3,4, sendo que abaixo de 3,0 ocorre uma tendência à sinérese. Em relação ao índice de acidez a geleia tradicional apresentou o menor valor (14,59). Sabe-se, no entanto, que a concentração hidrogeniônica e não a acidez titulável tem importância na geleificação, e que diferentes concentrações de sólidos solúveis requerem diferentes valores nas faixas de $\mathrm{pH}$ para uma completa geleificação (LICODIEDOFF, 2008).

Nas formulações diet e light os sólidos solúveis totais apresentaram ainda um valor reduzido 43,9 e 48 'Brix, respectivamente. Isso ocorreu devido à diminuição no tempo de cocção e redução/ausência de adição de sacarose ao produto. Resultados semelhantes foram encontrados por Granada et al.(2005) onde a formulação controle foi preparada com a concentração final de sólidos 
solúveis de $65^{\circ}$ Brix e as formulações light, com concentrações de sólidos solúveis entre 45-47 ${ }^{\circ}$ Brix.

\section{Análise Sensorial}

Dos participantes da pesquisa $48 \%$ eram do sexo feminino e $52 \%$ do sexo masculino, com idade entre 15 e 44 anos, $42 \%$ dos provadores possuem renda de 2 a 3 salários mínimos, e cerca de $50 \%$ apresentavam $2^{\circ}$ grau completo.

De acordo com resultados obtidos observou-se que não houve diferença estatística significativa entre as notas atribuídas (tabela 3), mesmo com evidente diferença na composição química entre as formulações. As notas atribuídas aos parâmetros sensoriais analisados indicam que os provadores gostaram de forma regular a moderada (conceitos "gostei razoavelmente" e "gostei muito") das diferentes geleias.

Tabela 3 - Resultados das médias, desvios-padrão e variância das notas atribuídas pelos provadores às diferentes formulações de geleia mista de maracujá com abacaxi

\begin{tabular}{ccccc}
\hline & \multicolumn{5}{c}{ Geleias } \\
\hline Atributos & Tradicional & Diet & Light & p-valor \\
Aparência & $7,14 \pm 1,31$ & $7,24 \pm 1,49$ & $7,26 \pm 1,37$ & 0,8985 \\
Aroma & $7,54 \pm 1,13$ & $7,20 \pm 1,23$ & $6,94 \pm 1,60$ & 0,0798 \\
Sabor & $7,60 \pm 1,37$ & $7,20 \pm 1,56$ & $7,26 \pm 1,40$ & 0,3322 \\
Textura & $6,86 \pm 1,67$ & $6,64 \pm 1,87$ & $7,12 \pm 1,59$ & 0,6224 \\
Impressão global & $7,60 \pm 1,05$ & $7,14 \pm 1,40$ & $7,36 \pm 1,19$ & 0,1715 \\
\hline
\end{tabular}

Resultados semelhantes foram encontrados por Tsuchiya et al. (2009) na elaboração de diferentes geleias de tomate utilizando hortelã e cravo da índia na composição, onde constatou-se que não houve diferença significativa ao nível de 5\% entre as geleias elaboradas, o mesmo ocorreu em estudo realizado por Filho (1995), com exceção ao atributo consistência, em geleias produzidas com extratos pectinosos do albedo, casca integral do maracujá e pectina cítrica comercial.

$\mathrm{Na}$ análise sensorial, observou-se que as maiores freqüências de notas entre 6 e 9 (gostei ligeiramente a gostei muitíssimo) foram das formulações tradicional, diet e light, tendo como soma das percentagens globais $91,6 \%, 86 \%$ e $91,2 \%$, respectivamente.

De acordo como índice de aceitabilidade, a geleia tradicional mostrou-se superior com $81,66 \%$, em seguida a light com 79,66\% e diet com 78,66\% (Figura 1). Segundo Chaves e Sproesser (2005) índices de aceitabilidade superiores a $70 \%$ indicam que o produto terá boa aceitação. 
Figura 1- Resultado do teste de aceitabilidade das diferentes formulações de geleia de maracujá, de acordo com os atributos avaliados em porcentagem de aceitação dos produtos

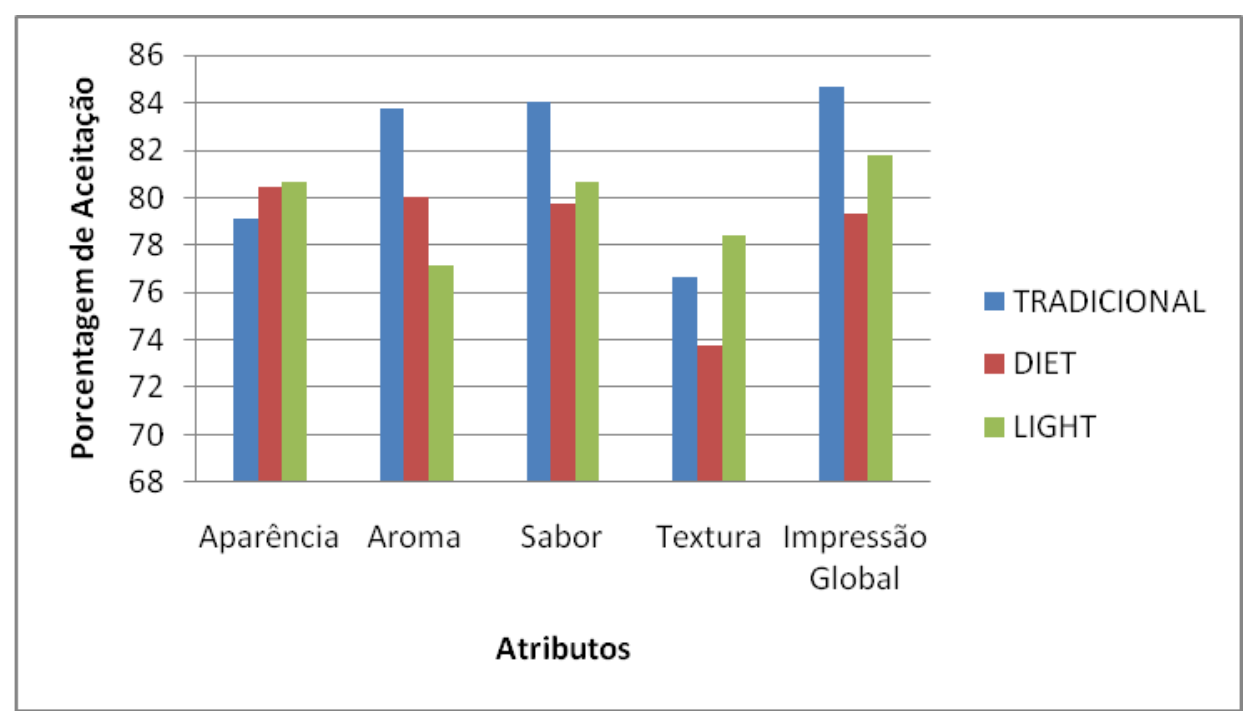

Na geleia tradicional os atributos sabor e aroma foram os mais apreciados pelos julgadores, demonstrando que a utilização de sacarose na formulação confere melhores características sensoriais, porém na aparência teve menor índice de aceitação $(79,11 \%)$, talvez por ter atribuído coloração mais escura a geleia.

O sabor residual conferido por edulcorantes é uma das principais dificuldades no desenvolvimento de produtos diet e light, porém a partir dos resultados obtidos em relação ao atributo sabor é possível notar que a diferença entre as formulações foi mínima, sendo assim a utilização de substitutos de açúcar mostrou-se aceitável na elaboração de geleias. Este fato corrobora com a discussão de Granada et al. (2005) que desenvolveu geleias light de abacaxi, demonstrando que não houve diferença significativa evidenciando grande similaridade entre as diferentes formulações quanto a este atributo.

Em relação à textura a formulação diet mostrou-se com menor índice de aceitação, devido à ausência de açúcar e a utilização de edulcorantes. Quando ocorre a retirada total do açúcar alteramse as características sensoriais do produto, tornando-se necessária a incorporação de um agente de corpo que mantenha a maior similaridade possível com as propriedades que a sacarose proporciona ao produto tradicional (RICHTER e LANNES, 2007). Segundo Freitas (2005) a sucralose é considerada como adoçante intensivo e não como agente de massa. Outro fator que pode ter interferido na textura seria o ponto de geleificação, pois em geleias dietéticas este depende do equilíbrio entre tipo e quantidade de pectina, $\mathrm{pH}$ e teor de sólidos do produto (NITZKE e MACHADO, 2004). Sabe-se que para uma boa geleificação em produtos com baixos teores de sólidos solúveis, há necessidade da utilização de pectina de baixa metoxilação, pois esta forma gel na ausência de sacarose, e, no entanto, a pectina encontrada naturalmente em frutas é de alta metoxilação exigindo teores elevados de sacarose (BARACAT e SCHMIDT, 2007; BIERHALZ, 
2010). Em relação à textura as demais formulações não diferiram significativamente entre si, pois a sacarose foi utilizada atuando como agente de corpo e no processo de geleificação.

Todas as formulações apresentaram um índice de aceitabilidade maior que $78 \%$, indicando aprovação entre os julgadores.

\title{
4 Conclusões
}

A utilização de albedo de maracujá em substituição à pectina permitiu a elaboração de geleias com boas características físico-químicas e sensoriais, contribuindo ainda com valores elevados de fibras totais nas geleias elaboradas. A utilização de edulcorantes em substituição parcial ou total à sacarose não interferiu significativamente nos atributos das geleias light e diet, sendo que estas obtiveram boa aceitação, em relação ao sabor, bem como a tradicional. As formulações apresentaram índices de aceitabilidade superiores a 78\%.

O uso da casca de maracujá para elaboração de geleias agrega valor nutricional ao produto e diminui ainda impactos ambientais.

\begin{abstract}
In the food sector share of raw material not used or discarded during processing are called waste. The processing of passion fruit juice industrialized generates tons of skins and seeds that can be used for preparing several products like jams. This work aimed to the preparation of jams mixed pineapple with passion fruit, including its hull, in different formulations (traditional, light and diet), for the use of waste food industries in addition to verifying the acceptability of the product. The jams were prepared at the Laboratory of Food Processing Technology, Faculty of Marilia-SP. Were prepared using pineapple species hawai (Smooth Cayenne), yellow passion fruit (Passiflora edulis Flavicarpa Degener), sucrose, glucose syrup and sweeteners, obtaining the final values of soluble solids of 65 Brix for traditional values and entre $48^{\circ}$ Brix and $43.9^{\circ}$ for light diet. It was evaluated the physico-chemical and sensory. All formulations showed an acceptance rate of greater than $78 \%$. As for the fiber content is found possible to indicate their intake in the prevention of gastrointestinal diseases, which suggests the sale of jams using passion fruit flesh pectin to replace.
\end{abstract}

Key-words: reuse of waste; fibers; new products; pineapple; soluble solids.

\section{Referências}

AYRES, M.; AYRES, J. R. M; AYRES, D, L.; SANTOS, A. S. 2005. BioEstat 5.0: aplicações estatísticas nas áreas das ciências biomédicas. Belém: Sociedade Civil Mamirauá. Brasília: Ministério da Ciência e Tecnologia; Belém: Imprensa Oficial do Estado do Pará, 324p.

BADAWI, C. Aproveitamento integral dos alimentos: melhor sobrar do que faltar?. Acadêmica de Nutrição da FSP USP. Maio, 2010.

BARACAT, C. A.; SCHMIDT, F. L. Extração de pectina atm do albedo da laranja por processo descontínuo. Faculdade de Engenharia de Alimentos - FEA, UNICAMP. 2007. 
BRASIL. Ministério da Saúde. Agência Nacional de Vigilância Sanitária. Alimentos com alegações de propriedades funcionais ou de saúde, novos alimentos/igredientes substancias bioativas e probióticos. Disponível em: <http://www.anvisa.gov.br/alimentos/comissoes/tecno_lista_alega.htm>. Acesso em mai. 2011.

BIERHALZ, A. C. K. Confecção e caracterização de biofilmes ativos à base de pectina btm e de pectina btm/alginato reticulados com cálcio. Campinas, 2010. 137 f. Dissertação (Mestrado em Engeharia Química)Departamento de Engenharia de Processos - Universidade Estadual de Campinas. 2010.

CAMARGO, P. et al. Redimento da pectina da casca do maracujá em seus estádios diferentes de maturação: verde, maduro e senescência. In: V SEMANA DE TECNOLOGIA EM ALIMENTOS. 21 a 25 de maio, 2007.Universidade Tecnológica Federal do Paraná - UTFPR - Ponta Grossa.

CÂNDIDO, L. M. B.; CAMPOS, A. M. Alimentos para fins especiais: dietéticos. São Paulo: Ed. Varela, 1996.

CASTRO, A. G. P.; FRANCO, L. J. Caracterização do consumo de adoçantes alternativos e produtos dietéticos por indivíduos diabéticos. Arquivo Brasileiro de Endocrinologia e Metabolismo, v. 46, n. 3, São Paulo, 2002.

CHAVES, J. B. P.; SPROESSER, R. L. Práticas de laboratório de análise sensorial de alimentos e bebidas. Viçosa: UFV, Imprensa Universitária. 2005.

CÓRDOVA, K. R. V.; GAMA, T. M. M. T. B.; WINTER, C. M. G.; KASKANTZIS NETO, G.; FREITAS, R. J. S. Características físico-químicas da casca do maracujá amarelo (Passiflora edulis flavicarpa degener) obtida por secagem. Boletim do Ceppa, v. 23, n. 2, 2005.

DERIVI, S. C. N.; MENDEZ, M. H. M. Uma visão retrospectiva da fibra e doenças cardiovasculares. São Paulo: Varela, 2001.

EVANGELISTA, J. Tecnologia em alimentos. São Paulo: Editora Atheneu, 2008.

FIGUEIREDO, L. P.; VAlENTE, W. A.; DiAs, M. V.; BORGES, S. V.; PEREIRA, P. A. P.; PEREIRA, A. G. T.; CLEMENTE, P. R. Efeito da adição de suco de maracujá e tempo de cozimento sobre a qualidade de doces do albedo de maracujá em calda. Ciência e Tecnologia de Alimentos, v. 29, n. 4, $2009 . \quad$ p. $840-846$. http://dx.doi.org/10.1590/S0101-20612009000400022

FILHO, J. F. L. Utilização da casca do maracujá amarelo (Passiflora edulis f. flavicarpa, Degener) na produção de geleia. Campinas, set. 1995. 22 f. Dissertação (Mestrado em Tecnologia em Alimentos) - Faculdade de Engenharia de Alimentos, Universidade Estadual de Campinas.

FRANCO, G. Tabela de composição química dos alimentos. 9 ed. SãoPaulo: Atheneu, 1993.

FREITAS, S. M. L.; Alimentos com alegação diet ou light: definições, legislação e implicações no consumo. São Paulo: Editora Atheneu, 2005.

GAVA, A. J. Princípios de tecnologia de alimentos . São Paulo: Editora Nobel, 2002.

GIUNTINI, E. B.; LAJOLO, F. M.; MENEZES, E. W. Potencial de fibra alimentar em países ibero-americanos: alimentos, produtos e resíduos. Archivos Latinoamericanos de Nutrición, Caracas, v. 53, n. 1, p. 1-7, 2003.

GULARTE, N. B. Manual de analise sensorial de alimentos. Pelotas: UFPEL, 2002. 59 p.

GRANADA, G. G; ZAMBIAZI, R. C; MENDONÇA, C. R. B. Abacaxi: produção, mercado e subprodutos. Boletim do CEPPA, v. 22, n. 2, p. 405-422, 2004.

GRANADA, G. G.; ZAMBIAZI, R. C.; MENDONÇA, C. R. B. ; SILVA, E. Caracterização física, química, microbiológica e sensorial de geleias light de abacaxi. Ciência e Tecnologia de Alimentos, v. 25, n. 4, p. 629-635, 2005. http://dx.doi.org/10.1590/S0101-20612005000400002

ISHIMOTO, F. Y.; HARADA, A. I.; BRANCO, I. G.; CONCEIÇÃO, W. A. S.; COUTINHO, M. R. Aproveitamento alternativo da casca do maracujá- amarelo (Passiflora edulis f. var.flavicarpadeg.) para produção de biscoitos. Revista Ciências Exatas e Naturais, v. 9, n. 2, 2007.

KROLOW, A. C. R. Preparo artesanal de geléias e geleiadas. Embrapa. Pelotas, RS. Jun, 2005. 
LAGO, E. S.; GOMES, E.; SILVA, R. Produção de geleia de jambolão (Syzygium cumini lamarck): processamento, parâmetros físico-químicos e avaliação sensorial. Ciência e Tecnologia de Alimentos, v. 26, n. 4, p. 847-852, 2006. http://dx.doi.org/10.1590/S0101-20612006000400021

LAMANTE, A. C. B.; DADA, M. A.; FURQUIM, M.; GRAVENA, C.; BELLARDE, F. B.; LUCIA, F. D. Obtenção de geleia “diet" elaborada com suco de maracujá. Revista Uniara, n. 16, p. 191-197, 2005.

LAZARIN, R. A. et al. Desenvolvimento de geleia de goiaba "diet" enriquecida com fibras. $5^{\circ}$ Congresso Interinstitucional de Iniciação Científica - CIIC, 2011.

LICODIEDOFF, S. Influência do teor de pectinas comerciais nas características físico-químicas e sensoriais da geleia de abacaxi. Universidade Federal do Paraná. Curitiba, 2008.

MAIA, S. M. P. C; Aplicação da farinha de maracujá no processamento do bolo de milho e aveia para fins especiais. Dissertação (Mestre em Tecnologia em Alimentos). 2007. 78 f. Universidade Federal do Ceará, Fortaleza 2007.

NACHTIGALL, A. M.; SOUZA, E. L.; MALGARIM, M. B. ; ZAMBIAZI, R. C. Geleias light de amora-preta. Boletim do CEPPA, v. 22, n. 2, p. 337-354, 2004.

NITZKE, J. A.; MACHADO, C. E. Desenvolvimento de geleia diet: aspectos tecnológicos. In: XVII CONGRESSO BRASILEIRO DE TECNOLOGIA DE ALIMENTOS.Universidade Federal do Rio Grande do Sul- UFRS 07 a 10 de Set., 2004.

OLIVEIRA, N. F.; NASCIMENTO, M. R. F.; BORGES, S. V; RIBEIRO, P. C. N.; RUBACK V. R. 'Aproveitamento alternativo da casca do maracujá-amarelo (Passiflora edulis F. Flavicarpa) para produção de doce em calda. Ciência e Tecnologia de Alimentos. v. 22, n. 3, p. 259-262, 2002. http://dx.doi.org/10.1590/S0101-20612002000300011

ORIANI, B. Desenvolvimento de um produto alimentício diet para pessoas diabéticas. In: VIII SIMPÓSIO DE ENSINO DE GRADUAÇÃO. Universidade Metodista de Piracicaba. Out., 2010.

ORTOLANI, B. G. et. al. Alimentos dietéticos. Faculdade de Ciências Farmacêuticas da Universidade de São Paulo Departamento de tecnologia bioquímica-farmacêutica. São Paulo. Set., 2008.

PINHEIRO, E. R. Pectina da casca do maracujá amarelo (Passiflora edulis flavicarpa): otimização da extração com ácido cítrico e caracterização físico-química. Universidade Federal de Santa Catarina. Florianópolis, 2007.

RICHTER, M. , LANNES S. C. S. Ingredientes usados na indústria de chocolates. Revista Brasileira de Ciências Farmacêuticas - Brazilian Journal of Pharmaceutical Sciences, v. 43, n. 3, 2007.

ROCKENBACH,C. Efeito hipoglicêmico de farinha de casca de maracujá (Passiflora edulisflavicarpa) em ratos. 2007. 30 f. Trabalho de Conclusão de Curso (Nutrição) - Faculdade Assis Gurgacz - FAG. Cascavel, 2007.

SALINAS, R. D. Alimentos e nutrição: introdução à bromatologia. 3 ed. Porto Alegre: Editora Artmed, 2002.

SOUZA, M. W. S; FERREIRA, T. B. O; VIEIRA, I. F. R. Composição centesimal e propriedades funcionais tecnológicas da farinha da cascado maracujá. Alimentos e Nutrição Araraquara. v. 19, n. 1, p. 33-36, 2008.

SPANHOLI, L.; OLIVEIRA, V. R. Utilização de farinha de albedo de maracujá (Passiflora edulis flavicarpa degener) no preparo de massa alimentícia. Alimentos e Nutrição, Araraquara. v. 20, n. 4, p. 599-603, out./dez. 2009

TSUCHIYA, A. C.; SILVA, A. G. M.; SOUZA, M.; SCHMIDT, C. A. P. Caracterização físico-química, microbiológica e sensorial de geleia de tomate. Revista Brasileira de Produtos Agroindustriais, v. 11, n. 2, p. 165$170,2009$.

Submetido em 05 juL 2011, Aceito para publicação em 04 out. 2012. 